\title{
Novel KIF7 Mutation in a Tunisian Boy with Acrocallosal Syndrome: Case Report and Review of the Literature
}

\author{
Aysegül Ibisler ${ }^{a}$ Ute Hehr ${ }^{b}$ Andre Barth ${ }^{c}$ Margarete Koch ${ }^{c}$ Jörg T. Epplen ${ }^{a}$ \\ Sabine Hoffjan ${ }^{\text {a }}$ \\ ${ }^{a}$ Department of Human Genetics, Ruhr University, Bochum, ${ }^{b}$ Center for and Department of Human \\ Genetics, University of Regensburg, Regensburg, and 'Children's Hospital Datteln, University Witten/Herdecke, \\ Datteln, Germany
}

\section{Key Words}

Acrocallosal syndrome - Agenesis of corpus callosum .

Ciliopathy $\cdot$ KIF7· Sonic hedgehog pathway

\begin{abstract}
Acrocallosal syndrome (ACLS) is a rare autosomal recessive disorder characterized by agenesis of the corpus callosum, facial dysmorphism, postaxial polydactyly of the hands as well as preaxial polydactyly of the feet, and developmental delay. Mutations in the KIF7 gene, encoding a molecule within the Sonic hedgehog $(\mathrm{SHH})$ pathway, have been identified as causative for ACLS but also for the fatal hydrolethalus syndrome and some cases of Joubert syndrome. We report here on a Tunisian boy who shows the clinical characteristics of ACLS and was found to have a novel homozygous KIF7 nonsense mutation. Further, we summarize the current knowledge about the clinical spectrum associated with KIF7 mutations as well as genetic and/or phenotypic overlap with ciliopathies and other mutations in the SHH pathway.
\end{abstract}

(c) 2015 S. Karger AG, Basel

Acrocallosal syndrome (ACLS; OMIM 200990) is a rare genetic disorder classified as ciliopathy and mainly characterized by agenesis of the corpus callosum, facial dysmorphism, postaxial polydactyly of the hands as well as preaxial polydactyly of the feet, and developmental delay [Putoux et al., 2011]. It was clinically described first by Schinzel [1979], who reported a 3-year-old male patient with a pattern of congenital malformations including macrocephaly, agenesis of the corpus callosum, hypertelorism, postaxial polydactyly of all limbs, duplication with syndactyly of the big toes, growth retardation, and gross motor and mental retardation [Schinzel, 1979]. One year later, a 2-year-old girl with a very similar clinical picture was reported suggesting 'acrocallosal syndrome' as designation for this condition and assuming autosomal dominant inheritance, since the 2 cases apparently occurred sporadically [Schinzel and Schmid, 1980]. However, additional (in part consanguineous) families with multiple affected individuals soon led to the assumption of autosomal recessive inheritance [Schinzel and Kaufmann, 1986]. Putoux et al. [2011] identified mutations in the KIF7 gene, coding for a member of the Sonic hedgehog (SHH) pathway (kinesin family member protein 7) as causative for ACLS. Additionally, KIF7 mutations were also found to be causative for the lethal autosomal recessively inherited hydrolethalus syndrome (HLS; OMIM 614120), which shares several features with ACLS [Putoux et al., 2011] and a subtype of Joubert syndrome (JBTS12; OMIM 200990) [Dafinger et al., 2011].

\section{KARGER 125}

(c) 2015 S. Karger AG, Base

$1661-8769 / 15 / 0064-0173 \$ 39.50 / 0$

E-Mail karger@karger.com

www.karger.com/msy
Aysegül Ibisler, MD

Department of Human Genetics, Ruhr University

Universitätsstrasse 150

DE-44801 Bochum (Germany)

E-Mail ayseguel.ibisler@ ruhr-uni-bochum.de 
Fig. 1. Clinical findings in the index patient at 6 months of age. A-C Dysmorphological facial features with prominent forehead, marked hypertelorism, low-set ears, short nose with anteverted nostrils, long prominent philtrum, posteriorly located small mandible and an open mouth with a protruding tongue. D, E Postaxial hexadactyly of the right foot and bifid hallux of the left foot.

Fig. 2. cMR imaging at the age of 4 months. A T2-weighted images showing agenesis of the corpus callosum, abnormal formation of the brain stem, hypoplasia of the cerebellar vermis and a large retrocerebellar cyst. B T1 transversal imaging without MTS.
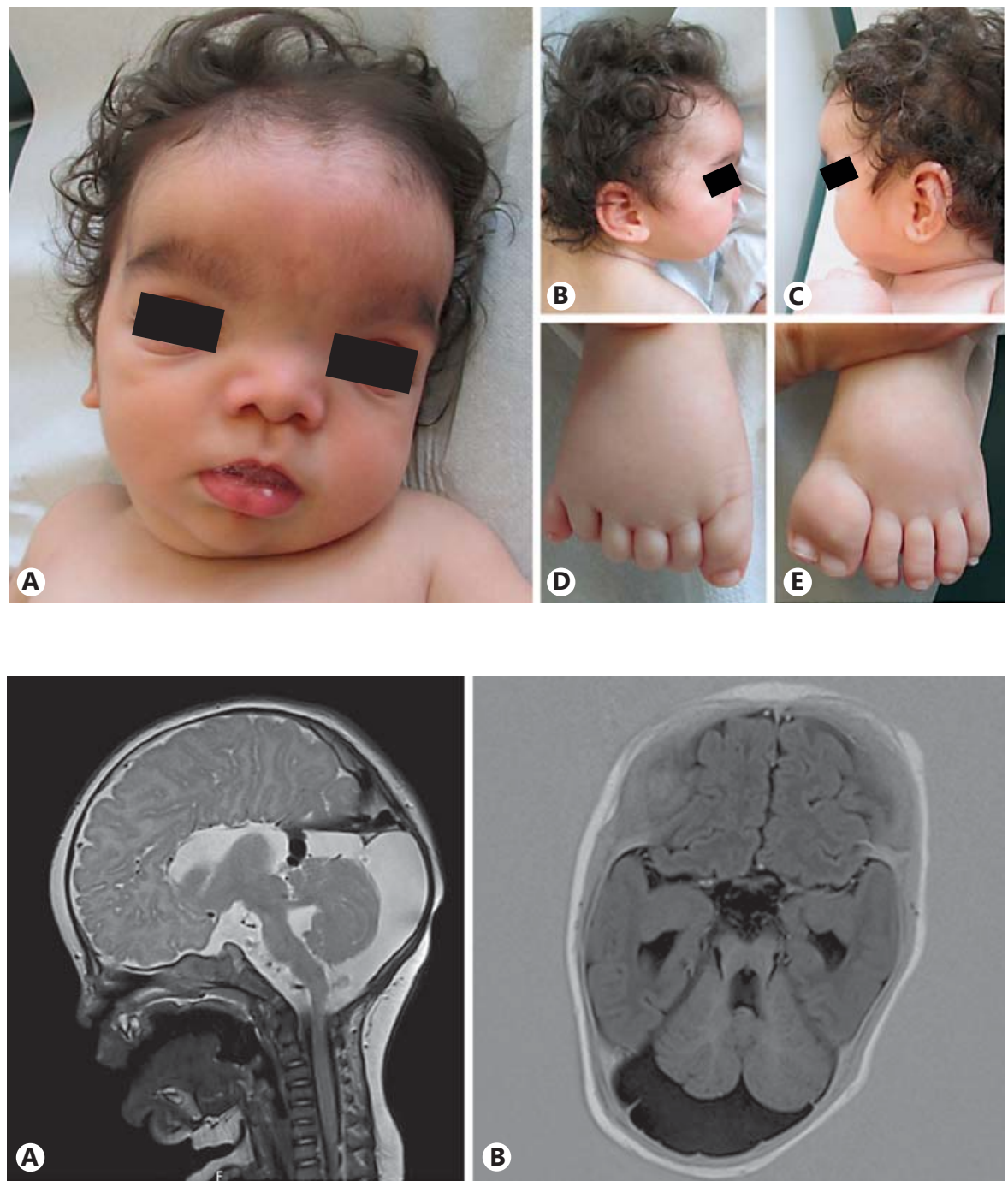

Here, we present a Tunisian boy with classical clinical characteristics of ACLS who carries a novel homozygous KIF7 nonsense mutation. In addition, we outline the current knowledge about the clinical features associated with KIF7 mutations as well as the role of KIF7 in the SHH pathway and limb development.

\section{Patient and Methods}

\section{Case Report}

The patient presented to the Department of Human Genetics of the Ruhr University, Bochum, at the age of 6 months. He is the second child of a healthy 26-year-old mother and a 31-year-old father, who decline consanguinity stemming from the same small village in Tunisia. The elder brother is healthy, and there is no family history of a syndromic disorder.

\section{Medical History}

The delivery of the patient occurred spontaneously at $38+4$ weeks of gestation after a normal pregnancy. Birth weight was $4,400 \mathrm{~g}$, length $57 \mathrm{~cm}$ and the head circumference was $38 \mathrm{~cm}$ (note macrosomia and macrocephaly with all parameters above the 97th percentile). The child showed respiratory distress syndrome symptoms with Apgar scores of 9, 6 and 3 after 1, 5 and 10 min, respectively, and a secondary reanimation was required. The following anomalies were noticed: large anterior fontanelle, hypertelorism and proptosis of the eyeball, retrognathia, postaxial polydactyly of the right foot and the right hand, and hallux duplication of the left food. Skull and heart ultrasounds as well as blood tests were performed, which revealed agenesis of the corpus callosum, hypoplasia of cerebellum, heart malformation with an aneurysm-like structure in the right ventricle and pericardial effusion as well as thrombocytopenia.

At the age of 4 days, the patient was transferred to another hospital with a department of pediatric cardiology, where the pericardial effusion was found to be hemodynamically insignificant. When 


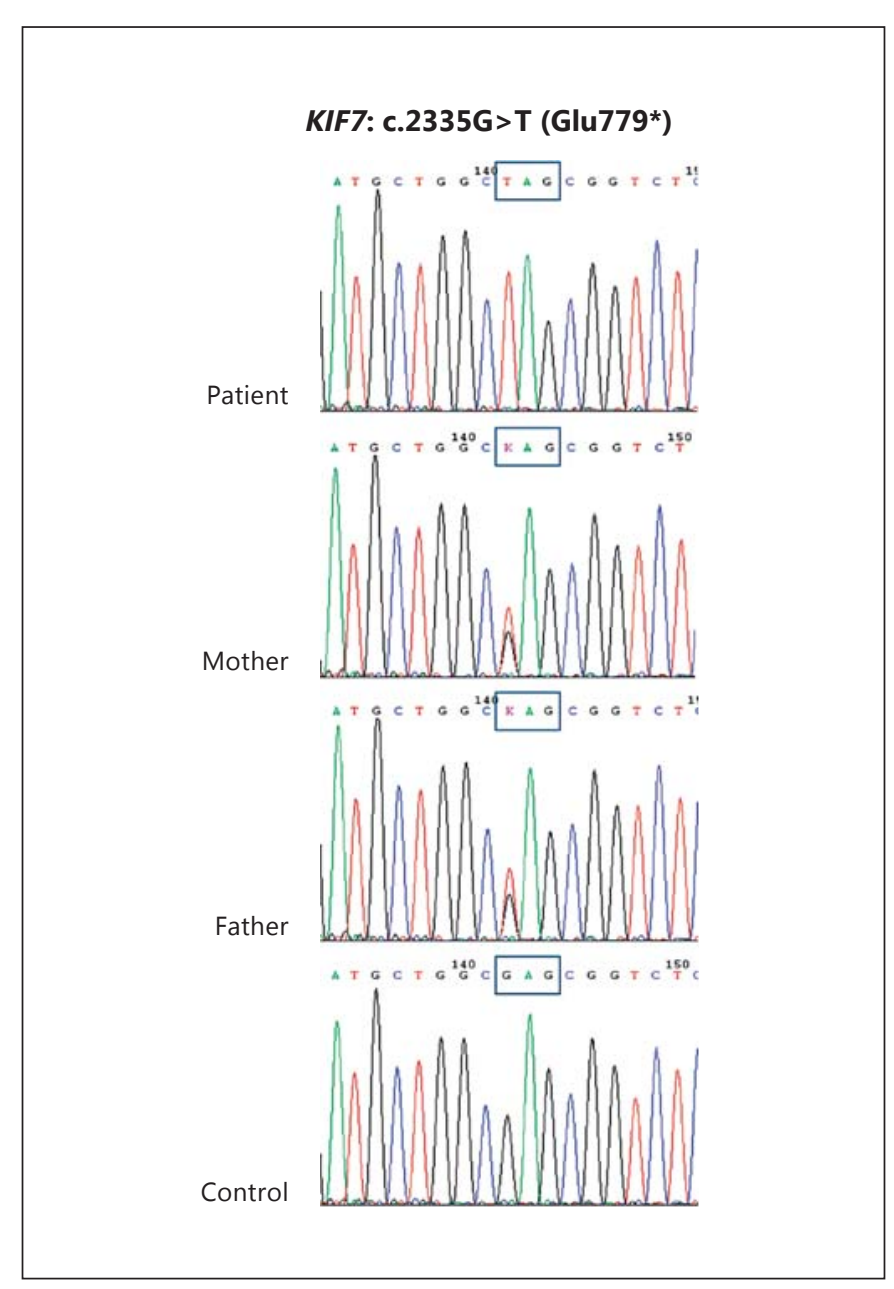

Fig. 3. Sequence analysis of the index patient, the parents and a control sample.

the patient was 2 months old, the rudimentary extra digit of the right hand was surgically removed. At the age of 3 months, he aspirated milk during feeding and needed to be resuscitated by his father. During in-patient treatment for aspiration pneumonia, the pediatricians initiated karyotyping and a SNP-array analysis (see below).

When the patient was 4 months old, he developed an epileptic seizure. The EEG analysis was compatible with focal increased susceptibility to seizures, and therefore, antiepileptic medication was initiated. Currently, at the age of 15 months, he shows severe global developmental delay with muscular hypotonia and only partial head control. He can roll over, but he is unable to crawl or sit unsupported. He often shows stereotypical behavior such as beating with his fists against his head. Length, weight and head circumference are now within the normal range for his age.

The family was referred to genetic counseling for a suspected syndromic disorder when the patient was 6 months old. Clinical evaluation at that time revealed dysmorphological facial features with a prominent forehead, marked hypertelorism, low-set ears, a short nose with anteverted nostrils, long prominent philtrum, pos-

KIF7 Mutation in a Boy with Acrocallosal Syndrome teriorly located small mandible, and an open mouth with a protruding tongue (fig. $1 \mathrm{~A}-\mathrm{C}$ ). At the lateral side of the fifth finger of the right hand, a surgical scar from amputation of the rudimentary extra digit was visible. Additionally, the patient showed a bifid hallux of the left foot and postaxial hexadactyly of the right foot (fig. 1D, E). He further exhibited generalized muscular hypotonia and unilateral maldescensus testis.

\section{MRI Findings}

Cerebral MRI was performed at the age of 4 months showing a plagiocephalic head configuration, absence of the corpus callosum with colpocephalic configuration of the lateral ventricles, a large retrocerebellar cyst as well as hippocampal malrotation and postnatal persistence of a zona along the medial walls of both lateral ventricles (fig. 2).

\section{Genetic Analyses}

Karyotype and SNP-array analysis had already been initiated by pediatricians. The karyotype was normal $(46, \mathrm{XY})$, and the SNP array did not reveal a pathogenetically relevant microdeletion or microduplication, but showed several regions with loss of heterozygosity (LOH; chromosomal regions 15q25.2q26.1, $16 \mathrm{p} 13.2 \mathrm{p} 12.3,16 \mathrm{p} 11.2 \mathrm{p} 11.1,16 \mathrm{q} 11.2 \mathrm{q} 12.2$ and $16 \mathrm{q} 21 \mathrm{q} 23.1)$, comprising $7 \%$ of the autosomal genome, which would correspond to consanguinity of first-degree cousins with a generation shift.

Since the clinical picture strongly pointed towards ACLS and the KIF7 gene is located in one of the regions (15q25.2q26.1) which showed LOH, we initiated Sanger sequencing of the KIF7 gene. This analysis identified a novel homozygous nonsense mutation in exon 11 of the KIF7 gene (c.2335G>T, p.Glu779*; fig. 3). Sequencing of the parents confirmed that they are both heterozygous carriers of this mutation.

\section{KIF7 Mutations in the Literature and Clinical Overlap with Other Diseases}

The KIF7 gene is located on chromosome $15 \mathrm{q} 26$ and comprises 19 exons (fig. 4). It contains a kinesin motor domain, a coiled-coil region and a cargo domain. Together with the novel mutation identified in the present study, 31 mutations have been described in this gene (HGMD ${ }^{\circledR}$ Professional, http://www.biobase-international. com/product/hgmd) [Barakeh et al., 2015; Karaer et al., 2015] to date which include nonsense, missense and splice site mutations as well as small deletions and span the entire gene without a hot spot region (fig. 4).

Putoux et al. [2011] described the first mutations in the KIF7 gene as causative for ACLS. Linkage and LOH analyses in 2 cohorts with a total of 8 ACLS cases identified an overlapping region of homozygosity on chromosome $15 \mathrm{q} 26$ in which the KIF7 gene was selected as the most promising candidate for sequence analysis due to its role in SHH signaling and limb formation (see below). All 8 ACLS patients were found to carry different homozygous 


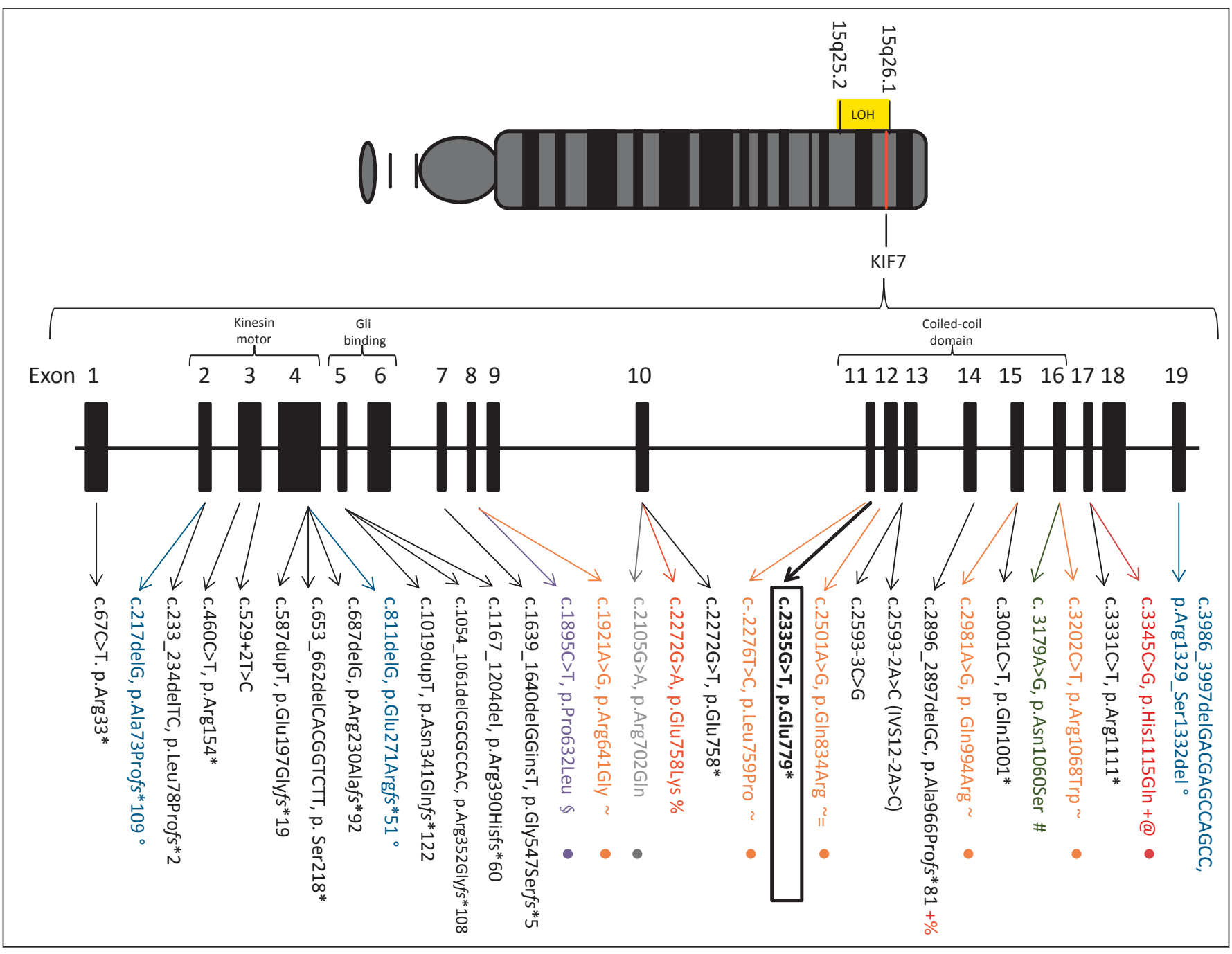

Fig. 4. Localization and structure of the KIF7 gene with the mutations described for different phenotypes in the literature as well as the present study. Mutations for ACLS are displayed in black. ${ }^{\circ}=$ Joubert syndrome; $\#$ = multiple epiphyseal dysplasia; + = hydrolethalus syndrome; $\%=$ intellectual disability; = Bardet-

truncating mutations in KIF7. They collectively presented with agenesis (6 cases) or hypoplasia ( 2 cases) of the corpus callosum, upper and/or lower limb polydactyly, intellectual disability, and diverse craniofacial dysmorphisms [Putoux et al., 2011]. After adding 5 ACLS patients with novel KIF7 mutations by the same group, however, a more variable expression of the phenotype emerged. While craniofacial dysmorphism was present in all 5 cases, one patient did not show a corpus callosum anomaly and another one did not exhibit polydactyly [Putoux et al., 2012]. Furthermore, compound heterozygous cases were presented for the first time. Following, a
Biedl syndrome; $\S=$ Pallister-Hall syndrome; $==$ orofaciodigital syndrome; @= Meckel syndrome. Filled circles indicate mutations that have only been observed in heterozygous state [Putoux et al., 2011]. Box: the novel mutation reported in the present study (c.2335G > T, p.Glu779*). consanguineous family with 5 affected members carrying a homozygous missense mutation in KIF7 was described that expanded the phenotypic spectrum [Ali et al., 2012]. The affected family members showed macrocephaly, an absent or hypoplastic corpus callosum and facial dysmorphism, but no polydactyly and only mild or absent intellectual disability. Instead, they exhibited skeletal abnormalities, especially multiple epiphyseal dysplasia [Ali et al., 2012]. Additional case reports have further added information to both the mutational and the clinical spectrum [Walsh et al., 2013; Hegde et al., 2015; Karaer et al., 2015], which may range from classical ACLS (as shown 
in the present case report) to only mild dysgenesis of the corpus callosum and intellectual disability without other associated features [Barakeh et al., 2015].

In the original report by Putoux et al. [2011], 6 of 8 ACLS patients showed a molar tooth sign (MTS) in the MRI, a feature which is often found in patients with Joubert syndrome (JS) [Romani et al., 2013], suggesting a clinical overlap between these 2 disease entities. In their second study, 3 of 5 patients again presented with MTS [Putoux et al., 2012]. JS and related disorders are a clinically and genetically heterogeneous group of disorders characterized by hypoplasia of the cerebellar vermis, with MTS as the characteristic neuroradiological finding and accompanying neurological symptoms such as a dysregulation of breathing pattern and developmental delay. Other variable features include retinal dystrophy and renal anomalies [Saraiva and Baraitser, 1992; Valente et al., 2013]. To date, 22 causative genes have been identified in JS and related disorders, and in accordance with the clinical overlap to ACLS, mutations in KIF7 were identified as causative for JS type 12 in a consanguineous Egyptian family [Dafinger et al., 2011]. It was suggested that MTS also is a frequent feature of ACLS, while corpus callosum agenesis is possible but rare in JS. In contrast, renal and retinal anomalies, which are often seen in JS, are absent in ACLS [Putoux et al., 2011, 2012]. Pathophysiologically, both ACLS and JS share an abnormal formation or function of cilia (see below) and have thus been classified as 'ciliopathies' [Waters and Beales, 2011].

KIF7 mutations in addition have been identified as causative for HLS, another autosomal recessive disorder sharing several features with ACLS but resulting in a more severe phenotype [Putoux et al., 2011]. It is a lethal embryonic condition characterized by hydrocephaly or anencephaly, postaxial polydactyly of the upper limbs and preor postaxial polydactyly of the lower limbs. Duplication of the hallux can also be observed. The first gene found to be associated with HLS is HYLS1, causing the phenotype HLS1 (OMIM 614120), while KIF7 causes HLS2 (OMIM 236680). Subsequently, all of these overlapping KIF7-associated syndromes (ACLS, JBTS12, HLS2, and the new phenotype associated with multiple epiphyseal dysplasia) have been suggested to constitute the phenotypic spectrum of KIF7-related ciliopathies [Putoux et al., 2012]; an overview of the major symptoms of these syndromes is given in table 1. So far, genotype-phenotype correlations are hard to establish; notably, one mutation has been found in homozygous state in a 14-year-old patient with ACLS, in a family with lethal HLS [Putoux et al., 2011] and in a boy only with intellectual disability [Barakeh et al., 2015].

KIF7 Mutation in a Boy with Acrocallosal Syndrome
For some ciliopathies, e.g. Bardet-Biedl syndrome, oligogenic inheritance has been suggested, which means that in addition to biallelic mutations in the respective primary disease gene, mutations in other ciliopathy genes may contribute to the phenotype [Walsh et al., 2013]. The same phenomenon was reported for an ACLS patient who carried a homozygous KIF7 mutation and additionally heterozygous loss-of-function variants in 3 other ciliopathy genes [Walsh et al., 2013]. Further, sequencing of KIF7 in patients with other ciliopathies found a significant enrichment for nonsynonymous heterozygous exchanges in this gene compared to controls [Putoux et al., 2011]. Thus, genetic interaction between different ciliopathy loci appears possible and may - at least to some extent - explain the variable phenotype associated with KIF7 mutations. Unfortunately, we were unable to test this hypothesis in our patient, since due to the classical phenotype only the KIF7 gene was sequenced.

There is also a significant clinical overlap of ACLS with Greig cephalopolysyndactyly syndrome (OMIM 175700), resulting from heterozygous GLI3 mutations. Greig cephalopolysyndactyly syndrome is characterized by polydactyly, widely spaced eyes and macrocephaly; less frequently agenesis of the corpus callosum and intellectual disability are observed [Démurger et al., 2015] (table 1). It was suggested that the milder end of the ACLS phenotypic spectrum may overlap with the severe end of the Greig cephalopolysyndactyly syndrome phenotype, and 2 patients clinically diagnosed with ACLS have been described to be heterozygous for a de novo GLI3 mutation [Elson et al., 2002; Speksnijder et al., 2013]. This remarkable clinical overlap is nicely reflected by a close functional relationship of KIF7 and GLI3, as they both serve as important signal transduction molecules within the SHH pathway [Anderson et al., 2012].

\section{Molecular Pathogenesis: The SHH Pathway and Limb Malformations}

The SHH pathway is a highly conserved signal transduction pathway important for numerous processes during embryonic development [Choudhry et al., 2014]. Among others, SHH signal transduction was shown to play a fundamental role in limb development [Anderson et al., 2012]. In this regard, the cilium as a specific structural component of the cell has come into focus. Cilia are microtubule-based structures, which are classified into 2 types: motile and immotile or primary cilia. In their center, they comprise a structure of microtubules (the so- 
Table 1. Differential diagnosis

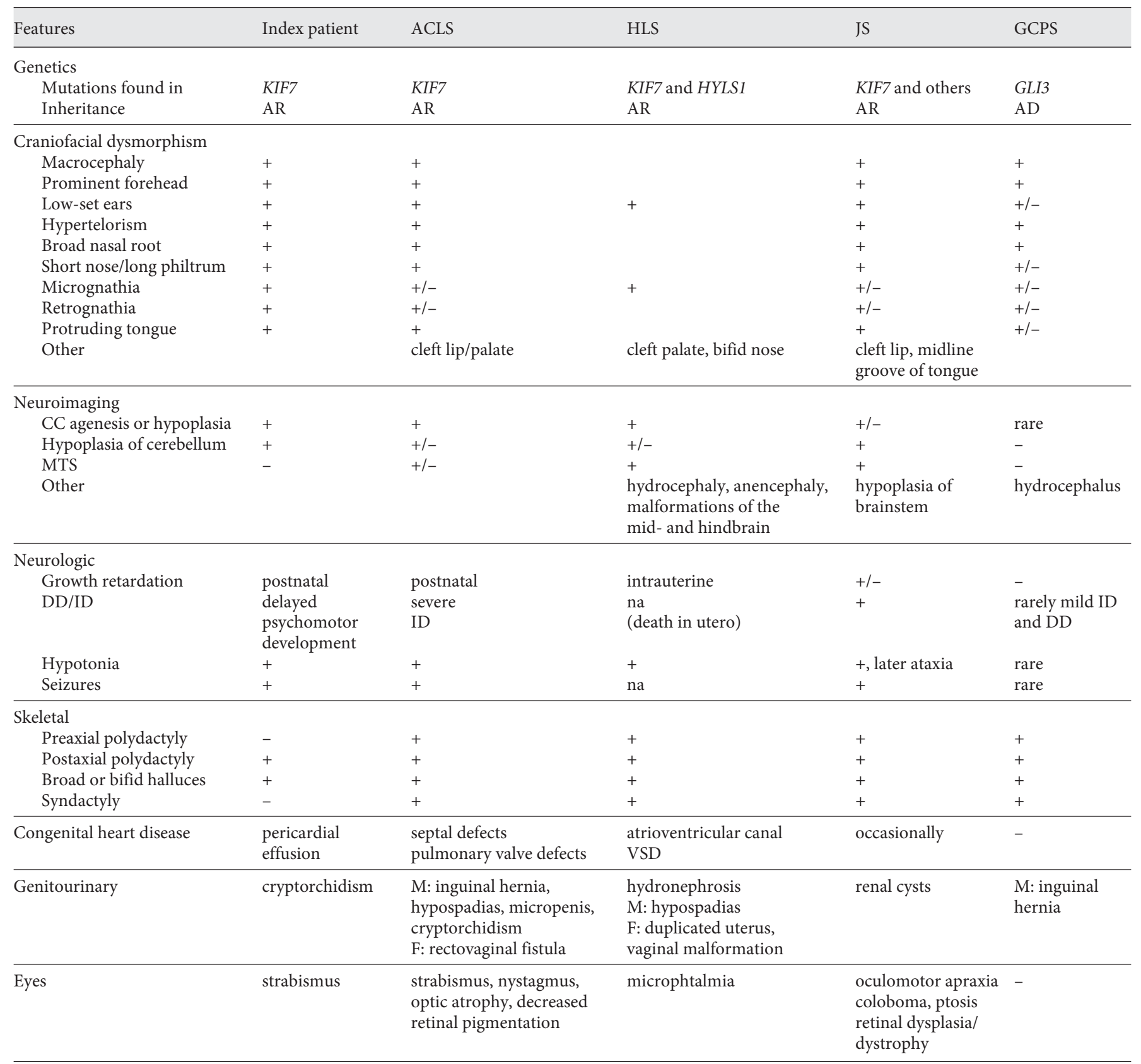

$\mathrm{AD}=$ Autosomal dominant; $\mathrm{AR}=$ autosomal recessive; $\mathrm{CC}=$ corpus callosum; $\mathrm{DD}=$ developmental delay; $\mathrm{F}=$ female; $\mathrm{GCPS}=\mathrm{Greig}$ cephalopolysyndactyly syndrome; $\mathrm{ID}=$ intellectual disability; $\mathrm{M}=$ male, na = not available; VSD = ventricular septal defect; + = reported; $-=$ not reported.

called axoneme) which is surrounded by a membrane lipid layer [Hildebrandt et al., 2011]. Proteins are transported along the axoneme, both in anterograde (towards the tip) and retrograde (towards the basis) direction; this mechanism has been called intraflagellar transport. Recent evidence suggested that intraflagellar transport com- ponents may be involved in SHH signaling [Anderson et al., 2012]. The GLI proteins (GLI2 and GLI3) are important transcription factors downstream of $\mathrm{SHH}$, which are transported along the axoneme (fig. 5). More specifically, in the absence of SHH binding to its ligand PTCH1, GLI3 is presumably kept in a complex with KIF7 and SUFU [He 


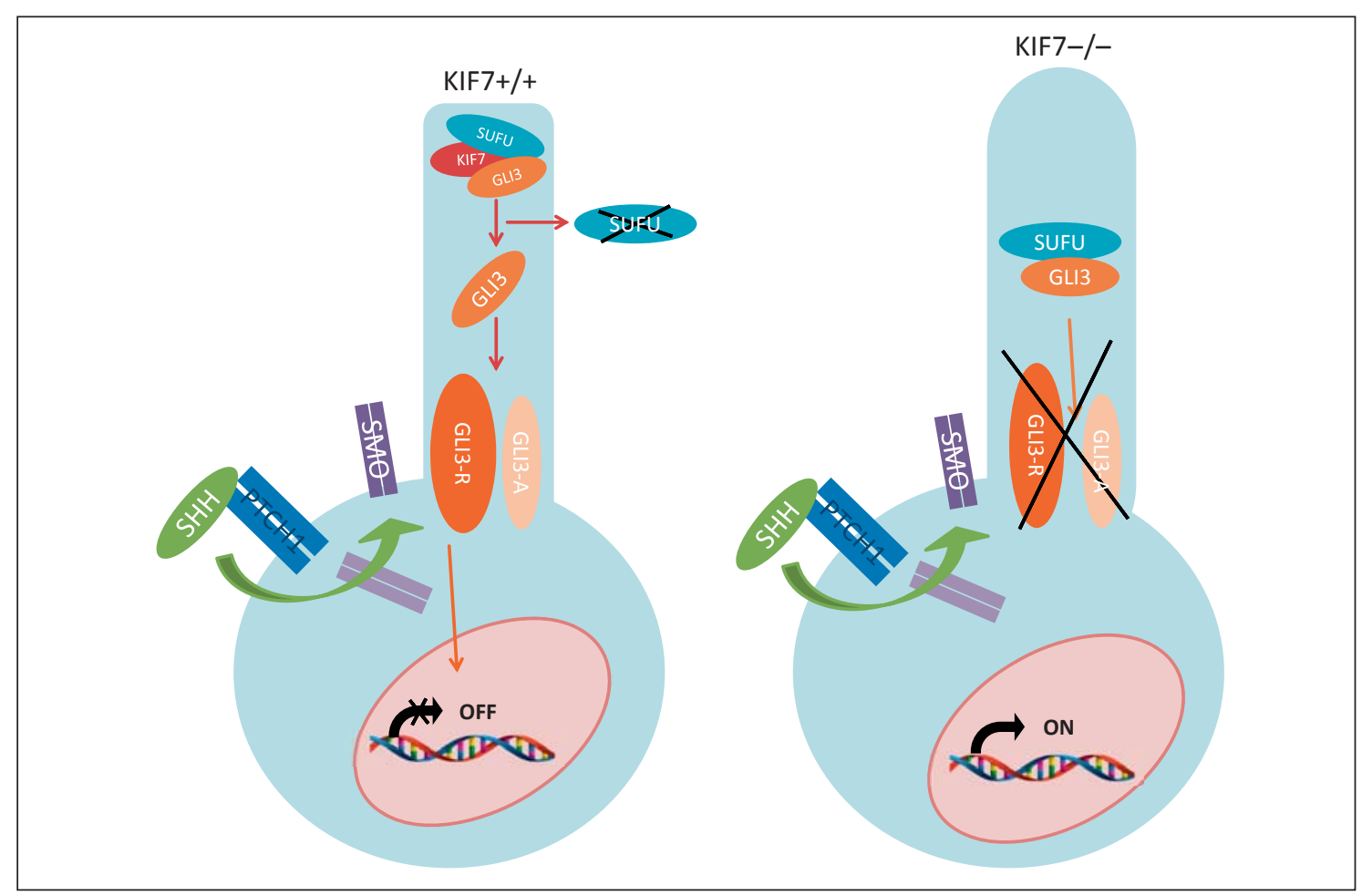

Fig. 5. SHH pathway in the cilium. In the absence of SHH, GLI3 is presumably kept in a complex with KIF7 and SUFU at the tip of the cilium and degraded to the repressive form (GLI3-R). Activation by SHH leads to translocation of the active form of GLI3
(GLI3-A) into the nucleus with subsequent transcription of respective target genes. In the absence of KIF7, the cilium is longer and repression of the transcription of target genes is abrogated. Adapted from Anderson et al. [2012]. et al., 2014; Pusapati and Rohatgi, 2014] at the tip of the cilium and subsequently degraded to the repressive form (GLI3-R). Activation by SHH, on the other hand, is supposed to release the inhibitory effect of SUFU and KIF7 and eventually leads to translocation of the active form of GLI3 (GLI3-A) into the nucleus with subsequent transcription of respective target genes [Anderson et al., 2012] (fig. 5). Furthermore, the important role of KIF7 and SUFU for SHH signaling and limb development has recently been demonstrated in an embryonic mouse model: while deletion of either KIF7 or SUFU resulted in the formation of ectopic digits (i.e. polydactyly), the combined loss of KIF7 and SUFU led to severe limb truncation [Zhulyn and Hui, 2015].

Polydactyly and anomalies of the cerebral midline including the pituitary gland and, less frequently, the corpus callosum may also result from heterozygous GLI2 mutations in humans [Roessler et al., 2003]. Polydactyly, cardiac defects and malformations of cerebral midline structures including agenesis of the corpus callosum or holoprosencephaly are also commonly observed in patients with Smith-Lemli-Opitz syndrome, an autosomal recessive metabolic disorder resulting from 7-dehydrocholesterol deficiency preventing the last step of cholesterol biosynthesis and its binding to $\mathrm{SHH}$ [Nowaczyk and Irons, 2012]. Moreover, gain-of-function mutations within an SHH regulatory element $1 \mathrm{Mb}$ upstream of $\mathrm{SHH}$, the zone of polarizing activity regulatory sequence, also cause a characteristic spectrum of limb malformations including polydactyly [Johnson et al., 2014].

\section{Conclusions}

In conclusion, an important role of KIF7 for SHH signaling and especially limb development has clearly been demonstrated over the last years, and phenotypic overlap exists with several disorders of the same pathophysiological pathway. The clinical spectrum associated with KIF7 mutations is wide, ranging from lethal HLS to mild dysgenesis of the corpus callosum and intellectual disability without other associated features, and it was suggested to group these phenotypes under 'KIF7-related ciliopathies'. As already shown for other cilia-related disorders such as 
Bardet-Biedl syndrome, genetic interaction between different ciliopathy loci appears possible and may - at least to some extent - explain the variable phenotype associated with KIF7 mutations. For complex and heterogeneous phenotypes such as many of the ciliopathies, nextgeneration sequencing techniques have proven to be a helpful tool, since they not only evaluate one or few single genes, but a whole group of suspected disease genes or the whole exome or even genome [Xing et al., 2014]. Even though both analytical and ethical issues will have to be solved before the latter techniques can become feasible on a routine basis [Davey, 2014], they will certainly lead to a better understanding of the complex mechanisms underlying ciliopathies and related disorders in the near future.

\section{Statement of Ethics}

Clinical and genetic evaluation of the presented patient was part of the routine diagnostic procedure. The parents of the patient gave their informed written consent to all investigations and publications.

\section{Disclosure Statement}

The authors state that they do not have any conflict of interests.

\section{References}

Ali BR, Silhavy JL, Akawi NA, Gleeson JG, AlGazali L: A mutation in KIF7 is responsible for the autosomal recessive syndrome of macrocephaly, multiple epiphyseal dysplasia and distinctive facial appearance. Orphanet J Rare Dis 7:27 (2012).

-Anderson W, Peluso S, Lettice LA, Hill RE: Human limb abnormalities caused by disruption of hedgehog signaling. Trends Genet 28:364373 (2012)

- Barakeh D, Faqeih E, Anazi S, Al-Dosari MS, Softah A, et al: The many faces of KIF7. HGV 2: 15006 (2015)

Brueton LA, Chotai KA, van Herwerden L, Schinzel A, Winter MW: The acrocallosal syndrome and Greig syndrome are not allelic disorders. J Med Genet 29:635-637 (1992).

-Choudry Z, Rikani A, Choudry AM, Tariq S, Zakaria $F$, et al: Sonic hedgehog signaling pathway: a complex network. Ann Neurosci 21: 28-31 (2014).

- Dafinger C, Liebau MC, Elsayed SM, Hellenbroich Y, Boltshauser E, et al: Mutations in KIF7 link Joubert syndrome and with Sonic Hedgehog Signaling and microtubule dynamics. J Clin Invest 121:2662-2667 (2011).

Davey S: Next generation sequencing: considering the ethics. Int J Immunogenet 41:457-462 (2014).

- Démurger F, Ichkou A, Mougou-Zerelli S, Le Merrer M, Goudefroye G, et al: New insights into genotype-phenotype correlation for GLI3 mutations. Eur J Hum Genet 23:92-102 (2015).

-Elson E, Perveen R, Donnai D, Wall S, Black GCM: De novo GLI3 mutation in acrocallosal syndrome: broadening the phenotypic spectrum of GLI3 defects and overlap with murine models. J Med Genet 39: 804-806 (2002).

-He M, Subramanian R, Bangs F, OmelchenkoT, Liem KF Jr, et al: The kinesin-4 protein Kif7 regulates mammalian Hedgehog signaling by organizing the cilium tip compartment. Nat Cell Biol 16:663-672 (2014).

Hegde DG, Mondkar J, Manerkar S: Acrocallosal syndrome with additional features in a neonate. J Clin Neonatol 4:51-53 (2015).

Hildebrandt F, Benzing T, Katsanis N: Ciliopathies. N Engl J Med 364:1533-1543 (2011).

Johnson EJ, Neely DM, Dunn IC, Davey MG: Direct functional consequences of ZRS enhancer mutation combine with secondary long range $\mathrm{SHH}$ signalling effects to cause preaxial polydactyly. Dev Biol 392:209-220 (2014).

Karaer K, Yuksel Z, Ichkou A, Calisir C, AttiéBitach T: A novel KIF7 mutation in two affected siblings with acrocallosal syndrome. Clin Dysmorphol 24:61-64 (2015).

Nowaczyk MJ, Irons MB: Smith-Lemli-Opitz syndrome: phenotype, natural history, and epidemiology. Am J Med Genet C Semin Med Genet 160C:250-262 (2012).

Pusapati GV, Rohatgi R: Location, location, and location: compartmentalization of Hedgehog signaling at primary cilia. EMBO J 33:18521854 (2014).

- Putoux A, Thomas S, Coene KLM, Davis EE, Alanay Y, et al: KIF7 mutations cause fetal hydrolethalus and acrocallosal syndromes. Nat Genet 43:601-606 (2011).

Putoux A, Nampoothiri S, Laurent N, CormierDaire V, Beales PL, et al: Novel KIF7 mutations extend the phenotypic spectrum of acrocallosal syndrome. J Med Genet 49:713-720 (2012).

Roessler E, Du YZ, Mullor JL, Casas E, Allen WP, et al: Loss-of-function mutations in the human GLI2 gene are associated with pituitary anomalies and holoprosencephaly-like features. Proc Natl Acad Sci USA 100:1342413429 (2003).

Romani M, Mocalizzi A, Valente EM: Joubert syndrome: congenital cerebellar ataxia with the molar tooth. Lancet Neurol 12:894-905 (2013).

Saraiva JM, Baraitser M: Joubert syndrome: a review. Am J Med Genet 43:726-731 (1992).

-Schinzel A: Postaxial polydactyly, hallux duplication, absence of the corpus callosum, macrencephaly and severe mental retardation: a new syndrome. Helv Paediat Acta 34:141-146 (1979).

Schinzel A, Kaufmann U: The acrocallosal syndrome in sisters. Clin Genet 30:399-405 (1986).

Schinzel A, Schmid W: Hallux duplication, postaxial polydactyly, absence of corpus callosum, severe mental retardation, and additional anomalies in two unrelated patients: a new syndrome. Am J Med Genet 6:241-249 (1980).

Speksnijder L, Cohen-Overbeek TE, Knapen MF, Lunshof SM, Hoogeboom AJ, et al: A de novo GLI3 mutation in a patient with acrocallosal syndrome. Am J Med Genet A 161A:13941400 (2013).

-Valente EM, Dallapiccola B, Bertini E: Joubert syndrome and related disorders. Handb Clin Neurol 113:1879-1888 (2013).

-Walsh DM, Shalev SA, Simpson MA, Morgan MA, Gelman-Kohan Z, et al: Acrocallosal syndrome: identification of a novel KIF7 mutation and evidence for oligogenic inheritance. Eur J Med Genet 56:39-42 (2013).

Waters AM, Beales PL: Ciliopathies: an expanding disease spectrum. Pediatr Nephrol 26: 1039-1056 (2011).

Xing DJ, Zhang HX, Huang N, Wu KC, Huang $\mathrm{XF}$, et al: Comprehensive molecular diagnosis of Bardet-Biedl syndrome by high-throughput targeted exome sequencing. PLoS One 9:e90599 (2014).

Zhulyn O, Hui CC: Sufu and Kif7 in limb patterning and development. Dev Dyn 244:468-478 (2015). 